\title{
The density of $S$-integral points in projective space with respect to a quadric
}

by

Nic Niedermowwe (Oxford)

1. Introduction. Let $S=\left\{p_{1}, \ldots, p_{m}, \infty\right\}$ be a finite set of places of $\mathbb{Q}$, including the archimedean one. The ring of $S$-integers is given by

$$
\mathcal{O}_{S}=\left\{y \in \mathbb{Q}:|y|_{p} \leq 1 \forall p \notin S\right\} .
$$

Its multiplicative subgroup $\mathcal{O}_{S}^{*}$ consists of the $S$-units, i.e. those elements of $\mathcal{O}_{S}$ whose $p$-adic absolute value equals 1 for all $p \notin S$. Given a smooth projective algebraic variety $X$ over $\mathbb{Q}$, and a smooth hypersurface $D \subset X$ defined by a form $F \in \mathbb{Z}\left[x_{1}, \ldots, x_{n}\right]$, we say that $\mathbf{x}=\left(x_{1}, \ldots, x_{n}\right) \in X$ is a $(D, S)$-integral point, or an $S$-integral point with respect to $D$, if $F(\mathbf{x}) \not \equiv 0$ $(\bmod p)$ for all $p \notin S$. We are interested in the asymptotic behaviour of the counting function

$$
\mathcal{N}(P)=\#\left\{(\mathbf{x}, Y) \in\left(\mathbb{Z}_{\text {prim }}^{n} \cap P B\right) \times \mathcal{O}_{S}^{*}: F(\mathbf{x})=Y\right\}
$$

for $(D, S)$-integral points of bounded height in the case where $X=\mathbb{P}^{n-1}$ and $F$ is a quadratic form. Here $P$ is a real parameter that tends to infinity, points in projective space are represented by primitive integral tuples, and $B$ is some $n$-dimensional hyperrectangle in $\mathbb{R}^{n}$ centred at the origin. In this form the problem corresponds to the degree two case of a question raised by Tschinkel [5, Problem 5.6].

Before we can give a precise statement of our main result, it is necessary to introduce some notation. We write $\mathcal{F}$ for the matrix of $F$ given by $F(\mathbf{x})=\frac{1}{2} \mathbf{x}^{T} \mathcal{F} \mathbf{x}$, and let $\mathcal{M}$ be a real orthogonal matrix that diagonalises $\mathcal{F}$. Accordingly, we choose $B$ such that the edges of $\mathcal{M}^{T} B$ are parallel to the coordinate axes. The set $\Lambda$ shall consist of all primes $p$ such that $p \mid 2 \operatorname{det} \mathcal{F}$ but $p \notin S$. We let $\Delta$ be the set of all $m+1$-tuples $\boldsymbol{\delta}$ with entries in $\{0,1\}$, and write $\mathbf{p}^{\boldsymbol{\delta}}=(-1)^{\delta_{0}} p_{1}^{\delta_{1}} \cdots p_{m}^{\delta_{m}}$ for short. For a given prime $p \in \Lambda \cup S$ and

2010 Mathematics Subject Classification: Primary 14G99; Secondary 11D45, 11D72, 14J99, $14 \mathrm{~N} 25$.

Key words and phrases: $S$-integral points, quadratic hypersurface, counting problem. 
$\boldsymbol{\delta} \in \Delta$ we define $t$ by $p^{t} \| 2 \mathbf{p}^{\delta}$. Finally, recall that for fixed $F$ a place is called (an) isotropic if $F$ does (not) represent zero non-trivially over the induced completion of $\mathbb{Q}$.

Theorem 1.1. Let $F, B$ and $S$ be given. Assume that $F$ is indefinite and $n \geq 4$. Then $\mathcal{N}(P)=0$ if for all $\boldsymbol{\delta} \in \Delta$ there exists $p \in \Lambda$ such that

$$
F(\mathbf{x}) \equiv \mathbf{p}^{\boldsymbol{\delta}}\left(\bmod p^{2 t+1}\right)
$$

has no solution. If there exists $\boldsymbol{\delta} \in \Delta$ such that the congruence is soluble for all $p \in \Lambda$, then

$$
\mathcal{N}(P) \sim c P^{n-2}(\log P)^{l} \quad \text { as } P \rightarrow \infty,
$$

where $c$ is some positive constant and $l$ is the number of non-archimedean isotropic places in $S$.

We attack the problem of counting the zeros of $Q(\mathbf{x}, Y)=F(\mathbf{x})-Y$ via the Hardy-Littlewood circle method. For non-zero integers $N$, it is a classical problem to find the number $R_{B}(P ; N)$ of zeros $\mathbf{x} \in P B$ of $Q(\mathbf{x}, N)$. Indeed, the theorem below can be proved using Kloosterman's refinement of the circle method (see e.g. 4]).

Theorem 1.2. For any $C>0$ one has

$$
R_{B}(P ; N)=I_{B}(P ; N) \mathfrak{S}(N)+O\left(P^{n-2}(\log P)^{-C}\right),
$$

where

$$
I_{B}(P ; N)=P^{n-2} \int_{-\infty}^{\infty} \int_{B} e\left(z Q\left(\mathbf{x}, N / P^{2}\right)\right) d \mathbf{x} d z
$$

and

$$
\mathfrak{S}(N)=\sum_{q=1}^{\infty} q^{-n} \sum_{\substack{a=1 \\(a, q)=1}}^{q} \sum_{\mathbf{x}(\bmod q)} e_{q}(a Q(\mathbf{x}, N))
$$

are the usual singular integral and singular series, respectively. The singular integral is convergent for all $N \in \mathbb{Z}$, and the singular series converges absolutely.

Since numbers representable by $F(\mathbf{x})$ with $\mathbf{x} \in P B$ have size $O\left(P^{2}\right)$ and there are $\asymp(\log P)^{m}$ such $S$-units (by the integrality of $F$ we may assume henceforth that $Y$ is restricted to values in $\mathcal{O}_{S}^{*} \cap \mathbb{Z}$ ), we obtain

$$
\mathcal{N}(P)=\sum_{Y \ll P^{2}} I_{B}(P ; Y) \mathfrak{S}(Y)+O\left(P^{n-2}(\log P)^{-1}\right) .
$$

This leads us to analyse the behaviour of the singular integral and series with respect to changing $Y$. The key observation is that if $p \in S$ and $F$ is anisotropic over $\mathbb{Q}_{p}$, then the local density for $p$ tends to zero as the power $p^{r}$ dividing $Y$ increases. Conversely, if $F$ is isotropic, i.e. represents 0 , over $\mathbb{Q}_{p}$, 
then the corresponding local density converges to a positive constant as $r$ grows. This is not surprising since as the power of $p$ dividing $Y$ increases, the $p$-adic size of $Y$ approaches zero.

The proof of Theorem 1.1 is completed in Section 4. By summing over the $S$-units as indicated above, the factors of $\log P$ in (2) are generated. It should be noted that $l=m$ if $n \geq 5$ since any quadratic form in at least five variables is isotropic over all $\mathbb{Q}_{p}$. If $n=4$ this is no longer true, and due to vanishing local densities any number of factors of $\log P$ may fail to occur. The exact shape of $c$ will become apparent and we obtain an error $O\left(P^{n-2}(\log P)^{l-1} \log \log P\right)$ in the asymptotic formula.

One should contrast Theorem 1.1 with the case of (positive) definite $F$ (and $k<0$ ). It is well-known that the total number of integral representations of a positive integer $N$ by $F$ is given by

$$
\frac{(2 \pi)^{n / 2}}{\Gamma(n / 2) \sqrt{\operatorname{det} \mathcal{F}}} \mathfrak{S}(N) N^{n / 2-1}+O\left(N^{(n-1) / 4+\varepsilon}\right)
$$

as $N \rightarrow \infty$. On using this formula in Section 4 , the reader can convince themselves that now $\mathcal{N}(P) \asymp P^{n-2}(\log P)^{l-1}$ if $(1)$ has a solution for all $p \in \Lambda$ and $l \geq 1$. If $l=0$ however, the singular series tends to zero. In fact, it dwindles rapidly enough to neutralise the growth of the singular integral, leaving us with an error term only for $\mathcal{N}(P)$. Indeed, since for anisotropic $p$ the form $F$ does not represent the integer $N$ if the greatest power of $p$ dividing $N$ is $\gg 1$, one easily sees that $\mathcal{N}(P)$ is constant for all sufficiently large $P$. Curiously, this constant may be zero as is illustrated by the example

$$
3\left(x_{1}^{2}+x_{2}^{2}\right)+14\left(x_{3}^{2}+x_{4}^{2}\right)-7^{r}=0 .
$$

There are no integral solutions to this equation although (1) is soluble for $p=2,3$ when $r=0$.

Lastly, we should note that almost everything done below goes through as soon as $n \geq 3$. Furthermore, at least since the work by Duke [2] and Iwaniec [3] on modular forms of half-integral weight it has been conjectured that Theorem 1.2 also holds when $n=3$. We will see in Section 3 that in this case the singular series is essentially a Dirichlet $L$-function evaluated at $s=1$, which diverges if and only if the character involved is principal. However, the singular series is originally a finite sum truncated at $P$, so that a simple pole at $s=1$ would lead to an extra factor of $\log P$ in (2).

Conjecture 1.3. Let $F, B$ and $S$ be given. Assume that $F$ is indefinite and $n=3$. Then $\mathcal{N}(P)=0$ if for all $\delta \in \Delta$ there exists $p \in \Lambda \cup S$ such that (1) has no solution. If there exists $\boldsymbol{\delta} \in \Delta$ such that (1) is soluble for all $p \in \Lambda \cup S$, then

$$
\mathcal{N}(P) \sim c P^{n-2}(\log P)^{l}, \quad c>0,
$$


if $-2 \mathbf{p}^{\boldsymbol{\delta}} \operatorname{det} F$ is not a perfect square for all such $\boldsymbol{\delta}$, and

$$
\mathcal{N}(P) \sim c P^{n-2}(\log P)^{m+1}, \quad c>0,
$$

otherwise.

Subject to the circle method succeeding, what we have said above in principle also applies to higher degree forms. Our observations highlight the dependence of the asymptotic behaviour of $\mathcal{N}(P)$ on the arithmetic of $F$.

Throughout we shall use $\varepsilon$ to represent any positive real number. No importance is attached to its exact size. Indeed, we allow different instances of $\varepsilon$ to take different values. Implicit constants in big- $O$ and $\ll$ notation may depend upon $F, B, S$ and $\varepsilon$.

2. The singular integral. Our first result shows that $I_{B}(P ; Y)$ is wellapproximated by $I_{B}(P ; 0)$ whenever $Y=o(P)$, and allows us to infer that the singular integral is of order of magnitude $O\left(P^{n-2}\right)$ for all $Y \ll P^{2}$.

Lemma 2.1. We have

$$
I_{B}(P ; Y)=I_{B}(P ; 0)+O\left(P^{n-2}\left(\left(|Y| / P^{2}\right)^{(n-2) / 5}+\left(|Y| / P^{2}\right)^{1 / 5}\right)\right) .
$$

Proof. On writing

$$
I_{B}(P ; Y)-I_{B}(P ; 0)=P^{n-2} \int_{-\infty}^{\infty} \int_{B} e(z F(\mathbf{x}))(e(z Y)-1) d \mathbf{x} d z
$$

we see that the part of the integration for which $|z|<R:=\left(|Y| / P^{2}\right)^{-2 / 5}$ is

$$
\ll \int_{|z|<R}\left|e\left(z\left(Y / P^{2}\right)\right)-1\right| d z \ll \frac{|Y|}{P^{2}} \int_{|z|<R}|z| d z \ll\left(|Y| / P^{2}\right)^{1 / 5} .
$$

Next we note that

$$
\int_{a}^{b} e\left(z v^{2}\right) d v \ll|z|^{-1 / 2}
$$

uniformly in $a, b \in \mathbb{R}$. This follows from observing that the integral over the range $\left[-|z|^{-1 / 2},|z|^{-1 / 2}\right] \cap[a, b]$ is trivially $\ll|z|^{-1 / 2}$, whence we may assume without loss of generality that $|z|^{-1 / 2} \leq a<b$. We then have

$$
\int_{a}^{b} e\left(z v^{2}\right) d v=\int_{a}^{b} 4 \pi i z v e\left(z v^{2}\right) \frac{1}{4 \pi i z v} d v \ll|z|^{-1 / 2}
$$

by partial integration. Therefore the contribution from $|z|>R$ to $I_{B}(P ; Y)$ is, after diagonalising $F$ via the substitution $\mathbf{x} \mapsto \mathcal{M} \mathbf{x}$,

$$
\ll\left(|Y| / P^{2}\right)^{(n-2) / 5} \text {. }
$$

The same applies for $I_{B}(P ; 0)$. 
The lemma above is complemented by the one below. Together they show that the singular integral is positive if $Y=o(P)$ and $P$ is sufficiently large.

LEMMA 2.2. Let $B$ be centred at a non-singular zero of $F$ and sufficiently small. Then

$$
I_{B}(P ; 0)=H_{B} P^{n-2}
$$

for some positive constant $H_{B}$.

Proof. This can be proved by an application of the implicit function theorem followed by an instance of Fourier inversion (compare for example Chapter 16 of [1]). In this context one only needs to note that any non-trivial zero $\mathbf{x} \in B$ of $F$ is necessarily non-singular.

3. The singular series. We begin our investigation of the singular series by performing the usual analysis which shows that it has an Euler product whose factors, the local densities

$$
\hat{\sigma}_{p}(Y)=\sum_{h=0}^{\infty} p^{-h n} \sum_{\substack{a=1 \\(a, p)=1}}^{p^{h}} \sum_{\mathbf{x}\left(\bmod p^{h}\right)} e_{p^{h}}(a Q(\mathbf{x}, Y)),
$$

can be expressed via the number of solutions to certain congruences. Subsequently we will see when the singular series vanishes and how it behaves with respect to changing $Y$.

LEMMA 3.1. We have

$$
\mathfrak{S}(Y)=\prod_{p} \hat{\sigma}_{p}(Y)
$$

Proof. For coprime integers $r$ and $s$ it is elementary to verify the multiplicative property

$$
S_{0}(r s, \mathbf{0})=S_{0}(r, \mathbf{0}) S_{0}(s, \mathbf{0}) .
$$

Together with the absolute convergence of the $\hat{\sigma}_{p}(Y)$ this proves that the Euler product representation of $\mathfrak{S}(Y)$ is valid.

A standard argument shows that the local densities satisfy

$$
\hat{\sigma}_{p}(Y)=\lim _{l \rightarrow \infty} p^{(1-n) l} \# \hat{M}\left(Y, p^{l}\right),
$$

where

$$
\hat{M}\left(Y, p^{l}\right)=\left\{\mathbf{x}\left(\bmod p^{l}\right): F(\mathbf{x}) \equiv Y\left(\bmod p^{l}\right)\right\} .
$$

This implies that the arithmetic functions $\hat{\sigma}_{p}$, and thus $\mathfrak{S}$, map into the non-negative real numbers.

Any $S$-unit is of the form $Y=p_{1}^{r_{1}} \cdots p_{m}^{r_{m}}$. Thus we shall say that $Y$ is of type $\boldsymbol{\delta}$ if

$$
\left((\operatorname{sign}(Y)-1) / 2, r_{1}, \ldots, r_{m}\right) \equiv \boldsymbol{\delta}(\bmod 2)
$$


For a given prime $p$ we define $\tau$ to be the integer that satisfies $p^{\tau} \| 2 Y$. Since there is a bijection from $\hat{M}\left(Y, p^{l}\right)$ to $\hat{M}\left(\mathbf{p}^{\delta} p^{2 k}, p^{l}\right)$, where $k=\lfloor\tau / 2\rfloor$ if $p$ is odd and $k=\lfloor(\tau-1) / 2\rfloor$ if $p=2$, the cardinality of $\hat{M}\left(Y, p^{l}\right)$ depends only on $\boldsymbol{\delta}, k$ and $l$. We shall make this explicit in our notation by setting

$$
M_{p}(\boldsymbol{\delta}, k, l)=\hat{M}\left(\mathbf{p}^{\boldsymbol{\delta}} p^{2 k}, p^{l}\right) \quad \text { and } \quad \sigma_{p}(\boldsymbol{\delta}, k)=\hat{\sigma}_{p}\left(\mathbf{p}^{\boldsymbol{\delta}} p^{2 k}\right) .
$$

Now Euler's identity $F(\mathbf{x})=\frac{1}{2} \mathbf{x} \cdot \nabla F(\mathbf{x})$ shows that for any $\mathbf{x} \in M_{p}(\boldsymbol{\delta}, k, l)$ with $l>\tau$ we have $p^{h} \| \nabla F(\mathbf{x})$ for some $h \in\{0, \ldots, \tau\}$. Therefore each $\mathbf{x} \in M_{p}(\boldsymbol{\delta}, k, l)$ falls into one of $\min (\tau+1, l+1)$ disjoint sets $M_{p}^{h}(\boldsymbol{\delta}, k, l)$ according to which

$$
h \in \begin{cases}\{0, \ldots, l-1, \infty\} & \text { if } l \leq \tau, \\ \{0, \ldots, \tau\} & \text { if } l>\tau,\end{cases}
$$

satisfies $p^{h} \| \nabla F(\mathbf{x})$.

LEMMA 3.2. If $h \neq \infty$ and $l \geq 2 h+1$, then

$$
\# M_{p}^{h}(\boldsymbol{\delta}, k, l+1)=p^{n-1} \# M_{p}^{h}(\boldsymbol{\delta}, k, l) .
$$

Proof. It is clear that the two sets $M_{p}^{h}(\boldsymbol{\delta}, k, l+i), i \in\{0,1\}$, can be partitioned into equivalence classes $E_{\mathbf{a}}^{i}$ according to the reduction a of their elements $\bmod p^{l-h}$. More precisely, as a disjoint union we have

$$
M_{p}^{h}(\boldsymbol{\delta}, k, l+i)=\bigcup_{\mathbf{a} \in A^{i}} E_{\mathbf{a}}^{i}
$$

where

$$
\begin{aligned}
E_{\mathbf{a}}^{i} & =\left\{\mathbf{x} \in M_{p}^{h}(\boldsymbol{\delta}, k, l+i): \mathbf{x} \equiv \mathbf{a}\left(\bmod p^{l-h}\right)\right\}, \\
A^{i} & =\left\{\mathbf{a}\left(\bmod p^{l-h}\right): \exists \mathbf{x} \in M_{p}^{h}(\boldsymbol{\delta}, k, l+i) \text { such that } \mathbf{a} \equiv \mathbf{x}\left(\bmod p^{l-h}\right)\right\} .
\end{aligned}
$$

First we shall show that

$$
\# E_{\mathbf{a}}^{0}=p^{h n} \quad \forall \mathbf{a} \in A^{0} .
$$

To see this fix an $\mathbf{a} \in A^{0}$ and choose an element $\mathbf{x} \in E_{\mathbf{a}}^{0}$. Now let $\mathbf{y}$ be any element $\left(\bmod p^{l}\right)$ with $\mathbf{y} \equiv \mathbf{a}\left(\bmod p^{l-h}\right)$. We can uniquely write $\mathbf{y}=$ $\mathbf{x}+p^{l-h} \mathbf{z}$ with $\mathbf{z} \in\left\{0, \ldots, p^{h}-1\right\}^{n}$. By linearity we have

$$
\nabla F(\mathbf{y})=\nabla F(\mathbf{x})+p^{l-h} \nabla F(\mathbf{z}),
$$

so that $p^{h} \| \nabla F(\mathbf{x})$ implies

$$
p^{h} \| \nabla F(\mathbf{y}) .
$$

Hence by Taylor-expanding we also have

$$
\begin{aligned}
F(\mathbf{y}) & \equiv F(\mathbf{x})+p^{l} \mathbf{z} \frac{\nabla F(\mathbf{x})}{p^{h}}+p^{2(l-h)} F(\mathbf{z})\left(\bmod p^{l}\right) \\
& \equiv F(\mathbf{x}) \equiv \mathbf{p}^{\boldsymbol{\delta}} p^{2 k}\left(\bmod p^{l}\right),
\end{aligned}
$$


which together with $(7)$ shows that $\mathbf{y} \in E_{\mathbf{a}}^{0}$. Since there are $p^{h n}$ possible $\mathbf{y}$, equation (6) holds.

Next we note that $A^{1} \subseteq A^{0}$. To see this, let $\mathbf{a} \in A^{1}$. Then there exists an $\mathbf{x} \in M_{p}^{h}(\boldsymbol{\delta}, k, l+1) \operatorname{such}$ that $\mathbf{x} \equiv \mathbf{a}\left(\bmod p^{l-h}\right)$. If we define $\mathbf{y}=\mathbf{x}\left(\bmod p^{l}\right)$ we have $\mathbf{x}=\mathbf{y}+p^{l} \mathbf{z}$ for some $\mathbf{z} \in\{0, \ldots, p-1\}$, whence the linearity of $\nabla F(\mathbf{x})$ gives $p^{h} \| \nabla F(\mathbf{y})$. It is now easily seen that $\mathbf{y} \in M_{p}^{h}(\boldsymbol{\delta}, k, l)$ and that $\mathbf{y} \equiv \mathbf{a}\left(\bmod p^{l-h}\right)$. Therefore $\mathbf{a} \in A^{0}$.

Finally we prove that

$$
\# E_{\mathbf{a}}^{1}=p^{h n+n-1} \quad \forall \mathbf{a} \in A^{0} .
$$

Fix an $\mathbf{a} \in A^{0}$ and consider any element $\mathbf{y}\left(\bmod p^{l+1}\right)$ with $\mathbf{y} \equiv \mathbf{a}\left(\bmod p^{l-h}\right)$. We uniquely write $\mathbf{y}=\mathbf{a}+p^{l-h} \mathbf{z}$ with $\mathbf{z} \in\left\{0, \ldots, p^{h+1}-1\right\}^{n}$. From (6) it follows that $\mathbf{a} \in E_{\mathbf{a}}^{0}$, which combined with the linearity of $\nabla F(\mathbf{y})$ shows that $p^{h} \| \nabla F(\mathbf{y})$. Moreover, we can expand

$$
F(\mathbf{y}) \equiv F(\mathbf{a})+p^{l} \mathbf{z} \frac{\nabla F(\mathbf{a})}{p^{h}}+p^{2(l-h)} F(\mathbf{z})\left(\bmod p^{l+1}\right) .
$$

Now $F(\mathbf{a})=\mathbf{p}^{\boldsymbol{\delta}} p^{2 k}+t p^{l}$ for some $t \in \mathbb{Z}$ since $\mathbf{a} \in M_{p}^{h}(\boldsymbol{\delta}, k, l)$, so that

$$
F(\mathbf{y}) \equiv \mathbf{p}^{\boldsymbol{\delta}} p^{2 k}\left(\bmod p^{l+1}\right)
$$

if and only if

$$
t+\mathbf{z} \frac{\nabla F(\mathbf{a})}{p^{h}} \equiv 0(\bmod p) .
$$

In the last congruence above, $\mathbf{z}$ is a solution if and only if $\mathbf{z}(\bmod p)$ takes one of exactly $p^{n-1}$ values since $p^{h} \| \nabla F(\mathbf{a})$, giving a total of $p^{h n+n-1}$ possible solutions z.

Now equation (9) implicitly gives the inclusion $A^{0} \subseteq A^{1}$, so that

$$
\begin{aligned}
\# M_{p}^{h}(\boldsymbol{\delta}, k, l+1) & =\sum_{\mathbf{a} \in A^{1}} \# E_{\mathbf{a}}^{1}=\# A^{0} p^{h n+n-1} \\
& =p^{n-1} \# A^{0} \# E_{\mathbf{a}}^{0}=p^{n-1} \sum_{\mathbf{a} \in A^{0}} \# E_{\mathbf{a}}^{0}=p^{n-1} \# M_{p}^{h}(\boldsymbol{\delta}, k, l),
\end{aligned}
$$

as claimed.

Corollary 3.3. We have

$$
\sigma_{p}(\boldsymbol{\delta}, k)=p^{1-n} \sum_{h=0}^{\tau} p^{-2 h(n-1)} \# M_{p}^{h}(\boldsymbol{\delta}, k, 2 h+1) .
$$

Proof. This follows immediately from the lemma.

If $p \notin S$, then $\tau$ is fixed, and the corollary implies that $\hat{\sigma}_{p}(Y)=\sigma_{p}(\boldsymbol{\delta}, 0)$ only depends on the type of $Y$. Furthermore, it transpires that for $p \in \Lambda$ the local density $\sigma_{p}(\boldsymbol{\delta}, 0)$ is positive if the congruence (1) is soluble. If $p \notin \Lambda \cup S$, 
then $\tau=0$ and there is the classical identity

$$
\sum_{\mathbf{x}(\bmod p)} e_{p}(a F(\mathbf{x}))=p^{n / 2} \varepsilon_{p}^{n}\left(\frac{2^{n} \operatorname{det} \mathcal{F}}{p}\right), \quad \varepsilon_{p}= \begin{cases}1 & \text { if } p \equiv 1(\bmod 4), \\ i & \text { if } p \equiv 3(\bmod 4),\end{cases}
$$

for the quadratic Gauss sum when $(2 a \operatorname{det} \mathcal{F}, p)=1$. Therefore

$$
M_{p}(\boldsymbol{\delta}, 0,1)=p^{n-1}+p^{n / 2-1} \varepsilon_{p}^{n}\left(\frac{\operatorname{det} \mathcal{F}}{p}\right) \sum_{a=1}^{p-1}\left(\frac{2 a}{p}\right)^{n} e_{p}\left(-a \mathbf{p}^{\boldsymbol{\delta}}\right),
$$

which by Corollary 3.3 yields

$$
\sigma_{p}(\boldsymbol{\delta}, 0)= \begin{cases}1-p^{-n / 2} \varepsilon_{p}^{n}\left(\frac{\operatorname{det} \mathcal{F}}{p}\right) & \text { if } n \text { is even and } \geq 2, \\ 1+p^{(1-n) / 2} \varepsilon_{p}^{n+1}\left(\frac{-2 \mathbf{p}^{\boldsymbol{\delta}} \operatorname{det} \mathcal{F}}{p}\right) & \text { if } n \text { is odd. }\end{cases}
$$

This shows that

$$
L_{\delta}=\prod_{p \notin S} \hat{\sigma}_{p}(Y)
$$

is a non-negative constant only dependent on the type of $Y$ if $n \geq 4$. It is zero if and only if the congruence (1) does not have a solution for some $p \in \Lambda$. With regard to our conjecture we observe that if $n=3$, then

$$
L_{\boldsymbol{\delta}}=\frac{L(1, \chi)}{L\left(2, \chi^{2}\right)} \prod_{p \notin S} \frac{\hat{\sigma}_{p}(Y)}{1+\chi(p) p^{-1}}
$$

equally depends only on the type of $Y$. Here $\chi$ is the Dirichlet character given by $\chi(p)=\left(-2 \mathbf{p}^{\boldsymbol{\delta}} \operatorname{det} \mathcal{F} / p\right)$; it is principal if and only if $-2 \mathbf{p}^{\boldsymbol{\delta}} \operatorname{det} \mathcal{F}$ is a square. Moreover, if (1) is soluble for all $p \in \Lambda \cup S$, then the quadratic form $f\left(x_{1}, \ldots, x_{4}\right)=F(\mathbf{x})-\mathbf{p}^{\boldsymbol{\delta}} x_{4}^{2}$ is isotropic over all $\mathbb{Q}_{p}$ by Lemma 3.2. When this coincides with $\chi$ being principal, then for any prime $p$ Hasse's invariant $\varepsilon_{p}(f)$ must satisfy

$$
\varepsilon_{p}(f)=(-1,-1)_{p},
$$

where $(\cdot, \cdot)_{p}$ is the Hilbert symbol relative to $\mathbb{Q}_{p}$. Because of the identities $\varepsilon_{p}(f)=\varepsilon_{p}(F)\left(-\mathbf{p}^{\boldsymbol{\delta}}, 2 \operatorname{det} \mathcal{F}\right)$ and

$$
(-1,-1)_{p}\left(-\mathbf{p}^{\boldsymbol{\delta}}, 2 \operatorname{det} \mathcal{F}\right)_{p}(-1,-2 \operatorname{det} \mathcal{F})_{p}=\left(\mathbf{p}^{\boldsymbol{\delta}},-2 \mathbf{p}^{\boldsymbol{\delta}} \operatorname{det} \mathcal{F}\right)=1,
$$

equation (11) is equivalent to $\varepsilon_{p}(F)=(-1,-2 \operatorname{det} \mathcal{F})_{p}$, which in turn implies that $F$ is isotropic over $\mathbb{Q}_{p}$.

If $p \in S$, then $\tau$ may grow with $P$. Subject to existence we define

$$
\rho_{p}=\lim _{k \rightarrow \infty} \sigma_{p}(\boldsymbol{\delta}, k)
$$

whose key properties are summarised as follows. 
THEOREM 3.4. The limit $\rho_{p}$ exists, is non-negative and independent of $\boldsymbol{\delta}$, satisfies

$$
\rho_{p}-\sigma_{p}(\boldsymbol{\delta}, k) \ll p^{-k},
$$

and equals zero if and only if $F$ is anisotropic over $\mathbb{Q}_{p}$.

Proof. Let us write

$$
S_{p}^{h}=\left\{\mathbf{x}\left(\bmod p^{2 h+1}\right): F(\mathbf{x}) \equiv 0\left(\bmod p^{2 h+1}\right) \wedge p^{h} \| \nabla F(\mathbf{x})\right\} .
$$

Since $F$ is non-singular, one has

$$
\# S_{p}^{h}, \# M_{p}^{h}(\boldsymbol{\delta}, k, 2 h+1) \leq \#\left\{\mathbf{x}\left(\bmod p^{2 h+1}\right): p^{h} \mid \nabla F(\mathbf{x})\right\} \ll p^{h n} .
$$

So setting

gives

$$
\rho_{p}=p^{1-n} \sum_{h=0}^{\infty} p^{-2 h(n-1)} \# S_{p}^{h}
$$

$$
\rho_{p}-\sigma_{p}(\boldsymbol{\delta}, k) \ll \sum_{h>\tau / 2} p^{-2 h(n-1)+h n} \ll p^{-k} .
$$

It is also clear now that $\rho_{p}=0$ if $F$ is anisotropic over $\mathbb{Q}_{p}$ since any element of $S_{p}^{h}$ can be lifted to a non-trivial $p$-adic zero by Lemma 3.2 . If $F$ does represent zero $p$-adically, then there exists a zero $\mathbf{x} \in \mathbb{Z}_{p}^{n}$ of $F$ with $p^{h} \| \nabla F(\mathbf{x})$ for some integer $h$. Therefore $\mathbf{x} \in S_{p}^{h}$, and $\rho_{p}$ must be positive.

4. Proof of Theorem 1.1. Without loss of generality we assume from now on that $p_{1}, \ldots, p_{l}$ are isotropic and $p_{l+1}, \ldots, p_{m}$ anisotropic. In the latter case we let

$$
\theta_{i}(\boldsymbol{\delta})=\sum_{s=0}^{\infty} \sigma_{p_{i}}(\boldsymbol{\delta}, s) .
$$

These series are convergent by Theorem 3.4. They are also positive since $F(\mathbf{x})=\mathbf{p}^{\boldsymbol{\delta}}$ has a solution over all $p$-adic fields. So if $p=p_{i}$, then multiplying such a solution by $p_{i}^{s_{i}}$ for a sufficiently large $s_{i}$ gives rise to an element of the set $M_{p_{i}}\left(\boldsymbol{\delta}, s_{i}, 4\left(s_{i}+1\right)\right)$, from which one deduces that $\sigma_{p_{i}}\left(\boldsymbol{\delta}, s_{i}\right)>0$.

Let $\mathcal{N}^{*}(P)$ be the counting function obtained by omitting the requirement that $\mathbf{x}$ is primitive from the definition of $\mathcal{N}(P)$. Since

$$
\mathcal{N}(P)=\sum_{Y} \mu(Y) \mathcal{N}^{*}(P / Y),
$$

an instance of Möbius inversion shows that it suffices to consider $\mathcal{N}^{*}(P)$. In analogy with (4) we have

$$
\mathcal{N}^{*}(P)=\sum_{\boldsymbol{\delta} \in \Delta Y \ll P^{2}} I_{B}^{\prime}(P ; Y) \mathfrak{S}(Y)+O\left(P^{n-2} K^{-1}\right),
$$

where the prime indicates that $Y$ is of type $\delta$, and $K=\log P$. 
By initially considering only that part of the summation which has $|Y| \leq$ $P^{2} K^{-5 m}$, we will be able to take advantage of Lemma 2.1. This lemma is fruitful only in combination with Lemma 2.2, whose requirements may not be satisfied by $B$. It is clearly possible though to choose some $n$-dimensional box $B_{0} \subset B$ that does satisfy the conditions of the lemma. One can then partition $B$ into a finite number of equally oriented $n$-dimensional boxes $B_{0}, B_{1}, \ldots, B_{g}$ which may or may not contain some or all of their faces. (Note that the absence of faces from the boxes $B_{i}$ is irrelevant with regard to the results proved in Section 2 , as the faces are nullsets in $\mathbb{R}^{n}$.) We obtain

$$
\sum_{Y \leq P K^{-5 m}}^{\prime} I_{B}(P ; Y) \mathfrak{S}(Y)=\sum_{Y \leq P K^{-5 m}}^{\prime} \sum_{i=0}^{g} I_{B_{i}}(P ; 0) \mathfrak{S}(Y)+O\left(P^{n-2} K^{-1}\right)
$$

since $\mathfrak{S}(Y) \ll 1$ by $(10)$. The definition of the singular integral in $(3)$ shows that

$$
I_{B_{i}}(P ; 0)=H_{B_{i}} P^{n-2}
$$

for some constant $H_{B_{i}}$. In addition, $H_{B_{i}}$ must be real and non-negative. For otherwise Theorem 1.2 applied to $N=\mathbf{p}^{\boldsymbol{\delta}} p_{1}^{2 k_{1}} \cdots p_{l}^{2 k_{l}} p_{l+1}^{2 s_{l+1}} \cdots p_{m}^{2 s_{m}}$, where the $s_{i}$ are fixed and defined as above and the $k_{i}$ tend to infinity with $P$, would, in combination with the resulting positivity of the singular series and Lemma 2.1, imply that there is a complex or negative number of zeros $\mathbf{x} \in P B_{i}$ of $Q(\mathbf{x}, N)$. Furthermore, $H_{0}>0$ since $B_{0}$ fulfils the conditions of Lemma 2.2. Therefore our summation becomes

$$
H P^{n-2} \sum_{|Y| \leq P^{2} K^{-5 m}}^{\prime} \mathfrak{S}(Y)+O\left(P^{n-2} K^{-1}\right),
$$

where $H=H_{0}+\cdots+H_{g}$ is a positive constant. Now let us write

$$
\sigma_{1}(Y)=\hat{\sigma}_{p_{1}}(Y) \cdots \hat{\sigma}_{p_{l}}(Y) \text { and } \sigma_{2}(Y)=\hat{\sigma}_{p_{l+1}}(Y) \cdots \hat{\sigma}_{p_{m}}(Y) .
$$

Then the summation in (14) is

$$
L_{\boldsymbol{\delta}} \rho_{p_{1}} \cdots \rho_{p_{l}} \sum_{|Y| \leq P^{2} K^{-5 m}}^{\prime} \sigma_{2}(Y)+O\left(\sum_{Y \leq P^{2} K^{-5 m}}^{\prime}\left|\sigma_{1}(Y)-\rho_{p_{1}} \cdots \rho_{p_{l}}\right| \sigma_{2}(Y)\right),
$$

where the error term is due to the convergence of the series $(13)$ bounded by

$$
\sum_{k_{1}, \ldots, k_{l} \ll K}\left|\sigma_{p_{1}}\left(\boldsymbol{\delta}, k_{1}\right) \cdots \sigma_{p_{l}}\left(\boldsymbol{\delta}, k_{l}\right)-\rho_{p_{1}} \cdots \rho_{p_{l}}\right| \ll K^{l-1} \log K .
$$

The final estimate above follows from splitting the summation according to whether or not $k_{i} \geq \log K$ for all $i=1, \ldots, l$, and then using the approximation $(12)$. We have shown that (14) is equal to

$$
H L_{\delta} \rho_{p_{1}} \cdots \rho_{p_{l}} P^{n-2} \sum_{|Y| \leq P^{2} K^{-5 m}}^{\prime} \sigma_{2}(Y)+O\left(P^{n-2} K^{l-1} \log K\right) .
$$


In what follows, we use the decomposition $Y=Y_{1} Y_{2}$ into factors $Y_{1}>0$ and $Y_{2}$ which contain the isotropic and anisotropic prime factors of $Y$ respectively. With this notation we can write the summation over the $Y$ as

$$
\sum_{Y_{1} \leq P^{2} K^{-5 m}}^{\prime} \sum_{Y_{2}}^{\prime} \sigma_{2}(Y)-\sum_{Y_{1} \leq P^{2} K^{-5 m}}^{\prime} \sum_{\left|Y_{2}\right|>P^{2} K^{-5 m} Y_{1}^{-1}}^{\prime} \sigma_{2}(Y),
$$

where the primes indicate that $Y_{1}$ is of type $\left(\delta_{1}, \ldots, \delta_{l}\right)$ and similarly for $Y_{2}$. Since the final summation above is bounded by

$$
\ll \sum_{\left|Y_{2}\right|>P^{2} K^{-5 m} Y_{1}^{-1}}\left|Y_{2}\right|^{-1} \ll\left(\frac{P^{2}}{K^{5 m} Y_{1}}\right)^{-1 / 2} \sum_{Y_{2}}\left|Y_{2}\right|^{-1 / 2} \ll\left(\frac{P^{2}}{K^{5 m} Y_{1}}\right)^{-1 / 2},
$$

we have

$$
\sum_{Y_{1} \leq P^{2} K^{-5 m}}^{\prime} \sum_{\left|Y_{2}\right|>P^{2} K^{-5 m} Y_{1}^{-1}}^{\prime} \sigma_{2}(Y) \ll P^{-1} K^{5 m / 2} \sum_{Y_{1} \leq P^{2} K^{-5 m}} Y_{1}^{1 / 2} .
$$

Now the sum above is $\ll P K^{-5 m / 2}$ if $l>0$ and equal to 1 otherwise. Therefore

$$
\sum_{|Y| \leq P^{2} K^{-5 m}}^{\prime} \sigma_{2}(Y)=\theta_{l+1}(\boldsymbol{\delta}) \cdots \theta_{m}(\boldsymbol{\delta}) \sum_{Y_{1} \leq P^{2} K^{-5 m}}^{\prime} 1+O\left(K^{l-1}\right),
$$

and it only remains to note that the $l$-dimensional pyramid given by the equations

$$
k_{i} \geq 0, \quad i=1, \ldots, l, \quad \text { and } \quad p_{1}^{\delta_{1}+2 k_{1}} \cdots p_{l}^{\delta_{l}+2 k_{l}} \leq P^{2} K^{-5 m}
$$

has volume

$$
\frac{1}{l !} \prod_{i=1}^{l} \frac{K}{\log p_{i}}+O\left(K^{l-1} \log K\right) .
$$

Via 15 and 14 we conclude that the contribution from $|Y| \leq P^{2} K^{-5 m}$ to $\mathcal{N}^{*}(P)$ is given by

$$
\frac{H L \rho_{p_{1}} \cdots \rho_{p_{l}}}{l ! \log p_{1} \cdots \log p_{l}} P^{n-2} K^{l}+O\left(P^{n-2} K^{l-1} \log K\right),
$$

where we set $L=\sum_{\boldsymbol{\delta} \in \Delta} L_{\boldsymbol{\delta}} \theta_{l+1}(\boldsymbol{\delta}) \cdots \theta_{m}(\boldsymbol{\delta})$.

Lastly, we need to consider the contribution from all $S$-units whose modulus is greater than $P^{2} K^{-5 m}$. By the results of the previous two sections this contribution clearly is

$$
\ll P^{n-2} \sum_{Y_{1} \leq P K^{-6 m}} \sum_{Y_{2} \geq K^{m}} \sigma_{2}(Y)+O\left(P^{n-2} K^{l-1} \log K\right) \ll P^{n-2} K^{l-1} \log K
$$

since the sum over all $Y_{2} \geq K^{m}$ is of size $O\left(K^{-1}\right)$ by Theorem 3.4 . 
If for all $\boldsymbol{\delta} \in \Delta$ there exists a prime $p \in \Lambda$ such that (1) has no solution, then obviously $\mathcal{N}(P)=0$. Otherwise $L>0$, and Theorem 1.1 holds with

$$
c=\frac{H L \rho_{p_{1}} \cdots \rho_{p_{l}}}{l ! \log p_{1} \cdots \log p_{l}} \prod_{p \in S}\left(1-p^{2-n}\right),
$$

where the product over the $p \in S$ stems from a Möbius inversion.

Acknowledgements. The author of this paper is studying for a D.Phil. at the University of Oxford. He would like to thank Prof. Heath-Brown for the excellent supervision that has accompanied this research.

\section{References}

[1] H. Davenport, Analytic Methods for Diophantine Equations and Diophantine Inequalities, 2nd ed., Cambridge Math. Library, Cambridge Univ. Press, Cambridge, 2005.

[2] W. Duke, Hyperbolic distribution problems and half-integral weight Maass forms, Invent. Math. 92 (1988), 73-90.

[3] H. Iwaniec, Fourier coefficients of modular forms of half-integral weight, ibid. 87 (1987), 385-401.

[4] N. Niedermowwe, Zeros of forms with $S$-unit argument, D.Phil. thesis, Univ. of Oxford, 2009.

[5] Y. Tschinkel, Geometry over non-closed fields, in: International Congress of Mathematicians (Madrid, 2006), Vol. II, Eur. Math. Soc., Zürich, 2006, 637-651.

Nic Niedermowwe

Mathematical Institute

24-29 St. Giles'

Oxford, OX1 3LB, United Kingdom

E-mail: niedermo@maths.ox.ac.uk

Received on 13.1.2009

and in revised form on 11.5.2009 